TRABAJOS ORIGINALES

Rev Obstet Ginecol Venez. 2021; 81 (1): 33-38

https://doi.org/10.51288/00810107

\title{
Detección de papilomavirus mediante reacción en cadena de la polimerasa en mujeres atendidas en el norte de Perú
}

\author{
Drs. (D) Sebastian Iglesias-Osores, ${ }^{1}$ (D) Luis Miguel Serquen-Lopez, ${ }^{1}$ Diana Saavedra-Muñoz, ${ }^{2}$ \\ Mariela LuzVásquez-Fernández, ${ }^{3}$ iD Tatiana Vidaurre. ${ }^{4}$
}

\section{RESUMEN}

Objetivo: El objetivo fue de determinar la incidencia de infección por infección por virus de papiloma humano en pacientes con citología desconocida mediante la técnica de reacción en cadena de la polimerasa en un hospital del norte de Perú.

Métodos: De las muestras de citocepillado de cérvix se extrajo el ADN por el método de extracción por sales (Salting Out). El ADN se extrajo y se amplificó mediante mediante reacción en cadena de polimerasa usando los primers MY09 y MY11.

Resultados: Se analizaron las muestras a 243 pacientes, el promedio de edad fue de 44 años. Mediante el uso de la técnica de reacción en cadena de la polimerasa (PCR) se encontró 13 pacientes $(5,35 \%)$ con resultado positivo para la presencia de virus papiloma humano, esto como parte de un programa de tamizaje en un hospital nivel III del norte del Perú.

Conclusión: La incidencia de virus papiloma humano fue baja. No hubo asociación entre grado de instrucción, estado civil, edad primera relación sexual y la infección por virus papiloma humano. No se encontró asociación entre número de parejas sexuales e infección por virus papiloma humano.

Palabras clave: Papiloma virus humano, Reacción en cadena de la polimerasa, Sondas de ADN de virus de papiloma humano, Infecciones por papillomavirus.

\section{SUMMARY}

Objective: The present study was conducted with the objective of determining the incidence of patients with human papillomavirus infection with unknown cytology using the polymerase chain reaction technique in a hospital in northern Peru.

Methods: DNA was extracted from cervical citobrush samples using the Salting Out method. The DNA that was extracted and amplified by polymerase chain reaction technique using primers MY09 and MY11.

Results: The samples were analyzed in 243 patients; the average age was 44 years. using the polymerase chain reaction technique, 13 patients (5.35\%) were found with a positive result for the presence of human papillomavirus, this as a part of a screening program in a Level III hospital in northern Peru.

Conclusions: The incidence of human papillomavirus was low. There was no association between educational level, marital status, age, first sexual intercourse and human papillomavirus infection. No association was found between the number of sexual partners and human papillomavirus infection.

Keywords: Human papilloma virus, Polymerase chain reaction, DNA probes, Papillomavirus Infections.

\section{INTRODUCCIÓN}

${ }^{1}$ Biólogo. Hospital Regional Lambayeque, Perú. ${ }^{2}$ Biólogo. Hospital Regional Lambayeque. Universidad Nacional Pedro Ruiz Gallo, Perú. ${ }^{3}$ Obstetra. Hospital Regional Lambayeque, Perú. ${ }^{4}$ Instituto Nacional de Enfermedades Neoplásicas.
El cáncer de cuello uterino se presenta como un importante problema de salud. Esta enfermedad constituye una de las entidades con más impacto y repercusión sobre la salud femenina, particularmente en las mujeres jóvenes, dada la alta morbilidad y mortalidad que produce (1). El cáncer de cuello 
uterino es la primera neoplasia que afecta a las mujeres en la región Lambayeque, Perú, y es la primera causa de mortalidad por cáncer en el Perú, encontrándose una frecuencia del $25 \%$ del total de neoplasias (2). Lambayeque se encuentra como la segunda región con el mayor índice de cáncer, después de Lima, en el Perú (3).

La infección por el virus del papiloma humano es un factor de riesgo para el desarrollo del cáncer de cuello uterino, varios estudios previos han caracterizado la epidemiología del virus del papiloma humano (VPH), donde la prevalencia de VPH de alto riesgo en mujeres adultas es del $12 \%$ y la prevalencia en cáncer de cuello uterino es $90 \%$ - $95 \%(4,5)$.

La presencia de VPH puede ser diagnosticada diez años antes de que se presenten síntomas, por consiguiente, el cáncer de cuello uterino es prevenible y curable (2). No obstante, la detección del cáncer cervical se realiza principalmente mediante una prueba de papanicolaou (citología convencional), técnica más utilizada para el despistaje de lesiones premalignas y malignas del cérvix uterino desde el siglo pasado. Sin embargo, la técnica presenta determinadas desventajas que hace que disminuya su efectividad y confiabilidad al analizar sus resultados, los tamizajes son ineficaces para detectar una infección temprana, porque la sensibilidad de la prueba es del 55,4\% y su especificidad del $96,8 \%$ en comparación con la detección molecular que muestra un $94,6 \%$ de sensibilidad y $94,1 \%$ de especificidad (6).

En Perú existe muy poca información y estudios sobre la incidencia de la infección por VPH, obtenida por técnicas moleculares. Se realizó el presente estudio con el objetivo de determinar la incidencia de infección por VPH en pacientes con citología desconocida mediante la técnica de reacción en cadena de la polimerasa (PCR) en un hospital nivel III del norte de Perú.

\section{MÉTODOS}

El presente trabajo fue descriptivo de corte transversal. La muestra estuvo conformada por todas las pacientes de ginecobstetricia del Hospital Regional Lambayeque, quienes acudieron a realizarse el papanicolaou en el periodo abril - junio de 2019. Se aplicó un consentimiento informado a las pacientes antes de la toma de muestra y un cuestionario donde se describió edad, grado de instrucción, estado civil, número de parejas sexuales. Se analizaron las muestras del cuello uterino, que fueron extraídas con un cepillo citológico en el consultorio de ginecobstetricia y procesadas en el Laboratorio de Biología Molecular del Hospital Regional Lambayeque. El protocolo de investigación tiene aprobación por el Comité de Ética del Hospital Regional Lambayeque.

\section{Detección de VPH por PCR}

Extracción de ácido desoxirribonucleico (ADN): las muestras se extrajeron con el citocepillo del cuello uterino de las pacientes y se usó el método de Salting Out para la extracción de ADN (7).

Reacción en cadena de la polimerasa: el ADN de las extracciones se amplificó mediante la técnica de PCR. Todas las muestras se amplificaron para las enzimas consenso de restricción de $450 \mathrm{pb}$ del gen L1 del VPH, MY09: (5'-CGTCCMARRGGAWACTGATC-3') y MY11: (5'-GCMCAGGGWCATAAYAATGG-3') (8-11), y se usó como control interno, hemoglobina subunidad beta (HBB) GH20: (5'-GAAGAGCCAAGGACAGGTAC-3') y PC04: (5'-CAACTTCATCCACGTTCACC-3') de $268 \mathrm{pb}$ (12). Si una muestra contenía ADN de betaglobina ( $\beta$-globina) detectable, entonces el ADN del VPH estaba intacto y amplificable durante la PCR (13).

Electroforesis y visualización de bandas: los 
productos amplificados fueron corridos en un sistema de electroforesis horizontal empleando gel de agarosa al 1,5\% usando un marcador de peso molecular de 100 pb y para su visualización se coloreó durante diez minutos con una solución de bromuro de etidio.

Procesamiento de datos y análisis estadístico: El procesamiento de geles se escaneó mediante el escáner molecular PharosFx Plus-BIORAD, las bandas fueron procesadas por el software Quantity one BIORAD (Análisis flexible de carriles y bandas, analizador de pureza y cuantificación rápida y fácil). Para identificar la asociación entre la infección por VPH y las características demográficas (edad, grado de instrucción, estado civil, número de parejas sexuales) se realizaron las pruebas T-student y Mann-Whitney utilizando el programa de Stata versión 15.

\section{RESULTADOS}

Se analizaron las muestras a 243 pacientes, en datos generales se encontró que el promedio de edad fue de

Tabla 1. Resultado de VPH de pacientes atendidas en el Hospital Regional Lambayeque según variables estudiadas

\begin{tabular}{lccc}
\hline & $\begin{array}{c}\text { Positivo } \\
\text { VPH } \\
\mathrm{n}(\%)\end{array}$ & $\begin{array}{c}\text { Negativo } \\
\text { VPH } \\
\mathrm{n}(\%)\end{array}$ & $\mathrm{p}$ \\
\hline Edad & $41(10,4)$ & $44,3(11,3)$ & 0,31 \\
Grado de instrucción & & & \\
Sin instrucción/primaria & $3(3,8)$ & $76(96,2)$ & 0,504 \\
Secundaria & $8(7,4)$ & $99(92,5)$ & \\
Superior & $2(3,5)$ & $55(96,5)$ & \\
Estado civil & & & \\
Soltera & $4(8)$ & $46(92)$ & 0,085 \\
Casada & $7(3,9)$ & $173(96,1)$ & \\
$\quad$ Separada & $2(15,4)$ & $11(84,6)$ & \\
Primera relación sexual & $18,69(4,3)$ & $18.63(4,0)$ & 0,53 \\
Parejas sexuales & $1(1)$ & $1(1)$ & 0,41 \\
\hline
\end{tabular}

44 años $(11,26 \%)$, el 32,51 \% (79/273) son pacientes sin instrucción/primaria, el 44,03\% (107/273) tiene educación secundaria y el 23,46\% (57/273) tiene educación superior. En cuanto al estado civil, un $20,58 \%(50 / 273)$ son solteras, un 74,07\% (180/273) son casadas y un 5,35\% (13) son separadas. La prueba de biología molecular para $\mathrm{VPH}$, fue positiva para el 5,35\% (13/273) y negativo para VPH el 94,65\% $(230 / 273)$.

No se encontró asociación entre grado de instrucción, estado civil, edad primera relación sexual y la infección de VPH. Según la prueba estadística Mann-Whitney no hay asociación entre número de parejas sexuales e infección por VPH (Tabla 1).

\section{DISCUSIÓN}

El presente trabajo se enfocó en la incidencia de la infección por VPH en muestras de cuello uterino de mujeres de la ciudad de Chiclayo, Lambayeque-Perú. Este trabajo confirmó una incidencia baja, ya que solo el 5,35\% de la muestra estudiada dio como resultado positivo para la prueba, más baja que la encontrada en otro estudio que fue del 12,6\% (14). Comparando los resultados con estudios en la región, difieren de un estudio chileno en el que se encontró 84,8 \% (15), en otro estudio en Argentina se encontró un 40 \% (16). Este resultado también difiere de un estudio realizado en la mismas región en la que se encontró un $23 \%$ (17).

En estudios peruanos se pueden encontrar resultados con una mayor incidencia, como del $23 \%$ (17), en estudiantes peruanas de Lima la frecuencia de VPH de alto riesgo fue 43,4\% (18), y en Cajamarca se encontró 30,5\% de casos positivos para VPH (19). En un estudio en Lima encontraron que 32,5\% (20) de los casos fueron VPH positivos, mientras que en una población andina se encontró el 20,5 \% (21).

La mayoría de las pacientes son casadas $(74,07 \%)$ y 
cuentan con educación secundaria completa $(44,03 \%)$. No se encontró asociación de estos factores con la detección de VPH, en contraste con estudios en que encuentran una fuerte asociación entre estos factores y la detección del virus $(22,23)$.

Se usó la técnica de PCR (24) convencional en este estudio pero existen numerosas técnicas para llegar al mismo resultado como Reverse Line-Blot (15) e inmunohistoquímica (25).

La edad de la primera relación sexual y la infección de VPH se encuentran muy asociadas, a más temprano inicio de la vida sexual, mayor probabilidad habrá de iniciar una infección por VPH, siendo este un factor de riesgo (26), seguido de los comportamientos sexuales (27). Esto difiere de los resultados del estudio porque no se encontró asociación entre estos factores. En el presente trabajo, no se encontró asociación entre el número de parejas sexuales y la infección por VPH; este resultado es contrario a los de otros estudios en los que existe relación entre el número de parejas sexuales y la infección por VPH (28) y su persistencia en la infección (29). El nivel de educación y pobreza ayuda para demostrar que un programa de tamizaje en un nivel III-1 no debe perderse, considerando que un nivel II no cuenta con equipos que permitan hacer pruebas de biología molecular, actualmente nivel III no puede hacer tamizaje y ello priva de hacerse esta prueba a más mujeres. Pacientes sin instrucción, si se acercan a los programas de tamizaje para VPH a un hospital nivel III-1, el programa Plan Esperanza funciona rompiendo barreras para un tamizaje molecular en una población $\mathrm{C}$ y D.

Existe una falta de estudios epidemiológicos de VPH en el Perú, sobre todo en poblaciones autóctonas. Se necesita saber qué factores influyen en la infección para poder diseñar campañas hacia el tamizaje. De esta manera, los estudios sobre VPH en zonas con diferentes tasas de incidencia de cáncer cervical contribuyen al conocimiento de la historia natural de la infección, de sus enfermedades asociadas y de los factores de riesgo, aportando información necesaria para el desarrollo de vacunas efectivas y el diseño de programas de prevención del cáncer cervical.

Se concluye que un 5,35\% de las pacientes estudiadas en un hospital de Chiclayo tienen infección por Papilomavirus.

Los autores agradecen a Virgilio E. Failoc-Rojas por su apoyo con el procesamiento estadístico del presente estudio.

Financiamiento: Plan Esperanza del Ministerio de Salud del Perú.

Conflicto de intereses: los autores declaran no tener conflictos de intereses.

\section{REFERENCIAS}

1. Siegel RL, Miller KD, Jemal A. Cancer statistics, 2018. CA Cancer J Clin. 2018; 68 (1):7-30. doi: 10.3322/ caac.21442. Epub 2018 Jan 4. PMID: 29313949.

2. Luciani, S, Winkler, J. Prevención del cáncer cervicouterino en el Perú: lecciones aprendidas del proyecto demostrativo tamizaje y tratamiento inmediato (TATI) de las lesiones cervicouterinas [Internet]. Washington: PAHO; 2006. [consultado 19 octubre 2017]. 1 p. Disponible en: http://www1.paho. org/spanish/ad/dpc/nc/pcc-cc-tati-rpt.pdf?ua=1

3. Ministerio de Salud [Internet]. Lima: Situación epidemiológica de la leishmaniasis, en el Perú. (Hasta la SE. No 52 2012) Boletín Epidemiológico; 2012 [consultado 19 octubre 2017];21 (52): 885-8. Disponible en: http://www.dge.gob.pe/boletines/2012/52.pdf

4. Aguilar A, Pinto JA, Araujo J, Fajardo W, Bravo L, Pinillos L, et al. Control of cervical cancer in Peru: Current barriers and challenges for the future. Mol Clin Oncol. 2016; 5 (2): 241-245. doi: 10.3892/ mco.2016.926. Epub 2016 Jun 10. PMID: 27446557; PMCID: PMC4950606. 
5. Bernal S. Detección y genotipado del virus del papiloma humano mediante una técnica de PCR a tiempo real: utilidad de la muestra de orina [Tesis doctoral en internet]. Sevilla (Andalucía): Universidad de Sevilla; 2014 [consultado 7 octubre 2017]. Disponible en: https://dialnet.unirioja.es/servlet/tesis? codigo $=71981$

6. Mayrand MH, Duarte-Franco E, Rodrigues I, Walter SD, Hanley J, Ferenczy A, et al. Human papillomavirus DNA versus Papanicolaou screening tests for cervical cancer. N Engl J Med. 2007; 357 (16): 1579-1588. doi: 10.1056/NEJMoa071430. PMID: 17942871.

7. Howe JR, Klimstra DS, Cordon-Cardo C. DNA extraction from paraffin-embedded tissues using a salting-out procedure: a reliable method for PCR amplification of archival material. Histol Histopathol [Internet]. 1997 [consultado 8 de marzo de 2019]; 12 (3): 595-601. Disponible en: http://www.ncbi.nlm.nih. gov/pubmed/9225139

8. Gravitt PE, Peyton CL, Alessi TQ, Wheeler CM, Coutlée F, Hildesheim A, et al. Improved amplification of genital human papillomaviruses. J Clin Microbiol [Internet]. 2000 [consultado 16 junio 2018]; 38 (1): 357-361. Disponible en: https://www.ncbi.nlm.nih. gov/pmc/articles/PMC88724/pdf/jm000357.pdf

9. Mendoza L, Arbiza J, Páez M, Kasamatsu E, Castro A, Giménez G, et al. Características clínico-demográficas y tipificación del virus de papiloma humano en mujeres paraguayas con citologías negativas para lesión escamosa intraepitelial. Mem Inst Investig Cienc Salud [Internet]. 2012 [consultado 7 octubre 2017]; 10 (1): 46-55. Disponible en: http://scielo.iics.una.py/pdf/iics/ v10n1/v10n1a06.pdf

10. Stamenković M, Knežević A, Knežević I, Kuzmanović I, Karalić D, Milenković S, et al. High-risk human papilloma virus genotypes in cervical carcinoma of Serbian women: Distribution and association with pathohistological findings. Biologicals. 2016; 44 (5): 412-416. DOI: 10.1016/j.biologicals.2016.05.001.

11. Mudini W, Palefsky JM, Hale MJ, Chirenje MZ, Makunike-Mutasa R, Mutisi F, et al. Human Papillomavirus Genotypes in Invasive Cervical Carcinoma in HIV-Seropositive and HIVSeronegative Women in Zimbabwe. J Acquir Immune Defic Syndr. 2018; 79 (1): e1-e6. doi: 10.1097/ QAI.0000000000001754. PMID: 29781877; PMCID: PMC6092204.

12. Reyna López L, González Cabeza J, Araujo Jiménez A, Ávila Vereau E, Gómez Castro K, Terán Rojas Y.
Detección y genotipificación de papiloma virus humano por PCR-RFLP en pacientes atendidas en el hospital distrital Walter Cruz Vilca La Libertad-2015. Pueblo Cont [Internet]. 2016 [consultado 7 octubre 2017]; 27 (2): 331-342. Disponible en: http://journal.upao.edu. pe/PuebloContinente/article/view/687.

13. Kleter B, van Doorn LJ, ter Schegget J, Schrauwen L, van Krimpen $\mathrm{K}$, Burger M, et al. Novel short-fragment PCR assay for highly sensitive broad-spectrum detection of anogenital human papillomaviruses. Am J Pathol. 1998; 153 (6): 1731-9. doi: 10.1016/ S0002-9440(10)65688-X. PMID: 9846964; PMCID: PMC1866345.

14. Nascimento M do DSB, Vidal FCB, Silva MACN da, Batista JE, Lacerda Barbosa M do C, Muniz Filho WE, et al. Prevalence of human papillomavirus infection among women from quilombo communities in northeastern Brazil. BMC Womens Health. 2018; 18 (1): 1. doi: 10.1186/s12905-017-0499-3.

15. Melo A, Vásquez AM, Andana A, Matamala M, Pino T, Guzmán P, et al. Genotipificación del virus papiloma humano en mujeres bajo 25 años de edad participantes del Programa Nacional del Cáncer Cérvico-uterino en la Región de la Araucanía, Chile. Rev Chil infectología [Internet]. 2014 [consultado 6 octubre 2017]; 31 (5): 542-8. Disponible en: http://www.scielo.cl/pdf/rci/ v31n5/art05.pdf.

16. Sijvarger CC, González J V, Prieto A, Messmer AG, Mallimaci MC, Alonio VL, et al. Epidemiología de la infección cervical por virus Papiloma humano en Ushuaia, Argentina. Rev Argent Microbiol [Internet]. 2006 [consultado 7 octubre 2017]; 38 (1): 19-24. Disponible en: http://www.redalyc.org/articulo. oa? id=213016797005.

17. Serquén-López LM, Iglesias-Osores SA, Arce-Gil ZL. Prevalencia de Papilomavirus Humano en trabajadoras sexuales atendidas en dos centros de salud de Chiclayo. Rev Cuerpo Médico HNAAA. 2017; 10 (4): 222-225. DOI: 10.35434/rcmhnaaa.2017.104.21

18. Manrique-Hinojosa J, Núñez-Teran MDC, PretelYdrogo L, Sullcahuaman-Allende Y, Roa-Meggo $\mathrm{Y}$, Juárez-Coello $\mathrm{P}$, et al. [Detection of the human papillomavirus in samples obtained by self-collection technique in a group of peruvian college students]. Rev Peru Med Exp Salud Publica. 2018; 35 (4): 642-646. Spanish. doi: 10.17843/rpmesp.2018.354.3450. PMID: 30726431.

19. Ponce-Benavente L, Rejas-Pinelo P, Aguilar-Luis MA, 


\section{DETECCIÓN DE PAPILOMAVIRUS MEDIANTE REACCIÓN EN CADENA}

DE LA POLIMERASA EN MUJERES ATENDIDAS EN EL NORTE DE PERÚ

Palomares-Reyes C, Becerra-Goicochea L, PinillosVilca L, et al. Frequency and coinfection between genotypes of human papillomavirus in a population of asymptomatic women in northern Peru. BMC Res Notes. 2018; 11 (1): 530. doi: 10.1186/s13104-0183644-7. PMID: 30064521; PMCID: PMC6069864.

20. Sullcahuaman-Allende Y, Castro-Mujica M, MejíaFarro R, Castaneda CA, Castillo M, Dolores-Cerna $\mathrm{K}$, et al. Características sociodemográficas de mujeres peruanas con virus papiloma humano detectado por PCR-RFLP. Rev Peru Med Exp Salud Publica [Internet]. 2015 [consultado 5 octubre 2017]; 32 (3): 509-514. Disponible en: http://www.scielosp. org/scielo.php?script=sci_arttext\&pid $=$ S172646342015000300015\&lng=en\&nrm=iso\&tlng=en

21. Silva-Caso W, Olivera-Irazábal M, León-Álvarez $\mathrm{P}$, Del Valle LJ, Díaz-Estacio S, Vargas M, et al. Identification of human papillomavirus as a preventive strategy for cervical cancer in asymptomatic women in the Peruvian Andes. Asian Pac J Trop Med. 2014; 7S1: S121-126. doi: 10.1016/S1995-7645(14)60217-0. PMID: 25312105.

22. Shakya S, Syversen U, Åsvold BO, Bofin AM, Aune G, Nordbø SA, et al. Prevalence of human papillomavirus infection among women in rural Nepal. Acta Obstet Gynecol Scand. 2017; 96 (1): 29-38. doi: 10.1111/ aogs.13036. Epub 2016 Nov 23. PMID: 27714759.

23. Franceschi S, Plummer M, Clifford G, de Sanjose S, Bosch X, Herrero R, et al. Differences in the risk of cervical cancer and human papillomavirus infection by education level. Br J Cancer. 2009; 101 (5): 865-870. doi: 10.1038/sj.bjc.6605224. Epub 2009 Aug 4. PMID: 19654578; PMCID: PMC2736843.

24. Sanoja LM. Detección y tipificación del virus del papiloma humano mediante reacción en cadena de polimerasa, en muestras cervicales de estudiantes. Comunidad y Salud [Internet]. 2013 [consultado 7 octubre 2017]; 11 (2): 1-10. Disponible en: http:// www.redalyc.org/html/3757/375740253002.
25. Marur S, D’Souza G, Westra WH, Forastiere AA. HPVassociated head and neck cancer: a virus-related cancer epidemic. Lancet Oncol. 2010; 11 (8): 781-9. doi: 10.1016/S1470-2045(10)70017-6. Epub 2010 May 5. PMID: 20451455; PMCID: PMC5242182.

26. León D, Retamal J, Silva R, Ili C, Mieville S, Guzmán $\mathrm{P}$, et al. Detección molecular de agentes infecciosos de transmisión sexual en un grupo de hombres sintomáticos y su relación con la conducta sexual. Rev Chil Infectol [Internet]. 2016 [consultado 7 octubre 2017]; 33 (5): 505-512. Disponible en: https://scielo.conicyt.cl/scielo.php? script $=$ sci arttext\&pid=S0716-10182016000500003

27. Kahn JA, Rosenthal SL, Succop PA, Ho GY, Burk RD. Mediators of the association between age of first sexual intercourse and subsequent human papillomavirus infection. Pediatrics. 2002; 109 (1): E5. doi: 10.1542/ peds.109.1.e5. PMID: 11773573.

28. Daling JR, Madeleine MM, Johnson LG, Schwartz SM, Shera KA, Wurscher MA, et al. Human papillomavirus, smoking, and sexual practices in the etiology of anal cancer. Cancer. 2004 Jul 15;101(2):270-80. doi: 10.1002/cncr.20365. PMID: 15241823.

29. Bosch FX, Lorincz A, Muñoz N, Meijer CJ, Shah KV. The causal relation between human papillomavirus and cervical cancer. J Clin Pathol. 2002; 55 (4): 244-65. doi: 10.1136/jcp.55.4.244. PMID: 11919208; PMCID: PMC1769629. 